Eastern Illinois University

The Keep

Faculty Research and Creative Activity

Physics

January 2008

\title{
Calculation of phonon dispersion in semiconductor nanostructures: An undergraduate computational project
}

Jie Zou

Eastern Illinois University, jzou@eiu.edu

Follow this and additional works at: http://thekeep.eiu.edu/physics_fac

Part of the Physics Commons

\section{Recommended Citation}

Zou, Jie, "Calculation of phonon dispersion in semiconductor nanostructures: An undergraduate computational project" (2008). Faculty Research and Creative Activity. 1.

http://thekeep.eiu.edu/physics_fac/1

This Article is brought to you for free and open access by the Physics at The Keep. It has been accepted for inclusion in Faculty Research and Creative Activity by an authorized administrator of The Keep. For more information, please contact tabruns@eiu.edu. 


\title{
Calculation of phonon dispersion in semiconductor nanostructures: An undergraduate computational project
}

\author{
Jie $Z{ }^{a}{ }^{a}$ \\ Department of Physics, Eastern Illinois University, Charleston, Illinois 61920
}

(Received 14 August 2007; accepted 24 November 2007)

\begin{abstract}
This paper discusses an undergraduate research project that involves the numerical calculation of phonon dispersion in semiconductor nanostructures. Phonon dispersion is calculated in the elastic continuum approximation by applying a finite-difference method to solving the elastic wave equation with boundary conditions. Students did simulations for two nanostructures: a free-standing GaN thin film and a free-standing AlN/GaN/AlN heterostructure. A comparison of these two structures helps students understand the effect of boundary conditions on the nature of the phonon dispersion. The project is related to current research in phonon thermal transport in semiconductor nanostructures and nanodevices. (C) 2008 American Association of Physics Teachers.
\end{abstract}

[DOI: 10.1119/1.2825396]

\section{INTRODUCTION}

Current technology in solid-state devices has reached the nanometer scale. Development of solid-state devices is driven by understanding of the novel properties and new physical phenomena in the underlying materials. Most solidstate devices, such as transistors, are based on semiconductors and their nanostructures. The advances in technology have stimulated much research interest among physicists and engineers in the properties of semiconductor nanostructures, such as quantum wells, quantum wires, and quantum dots. ${ }^{1}$ Although there has much work on the electronic properties of semiconductor nanostructures, research in phonon properties has attracted significant attention in recent years. ${ }^{2}$ Phonon engineering, an application analogous to electronic band-gap engineering, ${ }^{3}$ has recently been proposed, and provides new opportunities for controlling phonon transport in semiconductor nanostructures by tuning the phonon dispersion. In a semiconductor, heat is conducted mainly via lattice waves or phonons. In analogy to electronic band structure, phonon dispersion is an important property that directly affects the lattice thermal conductivity of the semiconductor. One of the main factors that drives the current interest in phonon heat conduction in semiconductor nanostructures is the increased heat dissipation associated with an increase in the transistor packing density.

Students in introductory solid-state physics often solve phonon dispersion problems in simple one-dimensional cases. ${ }^{4}$ Two typical examples are the monatomic and diatomic linear chain models, which can be solved analytically using lattice dynamics. ${ }^{4}$ Students are usually not familiar with three-dimensional problems and are not exposed to problems that require numerical solutions. Additionally, the results discussed in a typical undergraduate textbook apply only in bulk materials. Modifications are expected in a confined structure, such as a thin film (quantum well), a nanowire (quantum wire), and a heterostructure. In a semiconductor, such as silicon, heat conduction is due mainly to longwavelength acoustic phonons. ${ }^{5}$ An alternative approach to the calculation of phonon dispersion is the elastic continuum approximation, in which the equations of motion of the atoms are reduced to the continuum elastic wave equation. ${ }^{6,7}$

This paper discusses an undergraduate project in which students apply a finite-difference method to determine the phonon dispersion in a free-standing GaN thin film and a three-layer AlN/GaN/AlN heterostructure. The choice of $\mathrm{GaN}$ and its heterostructure is due to their potential use in high-power and high-temperature device applications. The goal of the project is to introduce students to current research topics in physics and engineering and to help them develop computational skills to solve more complicated problems. The project also helps students better understand the effect of boundary conditions on the characteristics of the phonon dispersion. Although the project focuses on phonons, the concept of quantization induced by boundary conditions applies equally well to an electron confined in a quantum well.

The rest of the paper is organized as follows. The next section presents the general theory of lattice thermal conductivity in order to illustrate the importance of phonon dispersion. Section III presents the continuum elastic wave equations, followed by a discussion in Sec. IV on the numerical algorithm for solving phonon dispersion. The simulation results are discussed in Sec. V.

\section{LATTICE THERMAL CONDUCTIVITY AND PHONON DISPERSION}

The law of thermal conduction can be found in most introductory texts. ${ }^{8}$ In the one-dimensional case, it takes the form, $J=\kappa|d T / d x|^{8}$, where $J$ is the heat flux or the thermal energy transmitted per unit cross-sectional area per unit time, and $|d T / d x|$ is the magnitude of the temperature gradient. The proportionality constant $\kappa$ is the thermal conductivity. More generally, $d T / d x$ should be replaced by the temperature gradient $\nabla T$. The heat flux $J$ can be expressed in terms of phonons as a sum over contributions from all phonon modes: ${ }^{9}$

$$
J=\sum_{\mathbf{k}, \alpha} n_{\mathbf{k}, \alpha} \hbar \omega_{\alpha}(\mathbf{k}) v_{\alpha}(\mathbf{k})_{\nabla T}
$$

A phonon mode is specified by the wave vector $\mathbf{k}$ and the phonon branch $\alpha . n_{\mathbf{k}, \alpha}$ is the number of phonons of type $(\mathbf{k}, \alpha)$ per unit volume of the crystal. The phonon angular frequency is given by $\omega$, and the energy of a phonon of type $(\mathbf{k}, \alpha)$ is given by $\hbar \omega_{\alpha}(\mathbf{k}) . v_{\alpha}(\mathbf{k})_{\nabla T}$ is the component of the phonon group velocity in the direction of the temperature gradient. Equation (1) for the heat flux is analogous to that for the electric current density, in which electron density, charge, and drift velocity are the counterparts of the phonon 


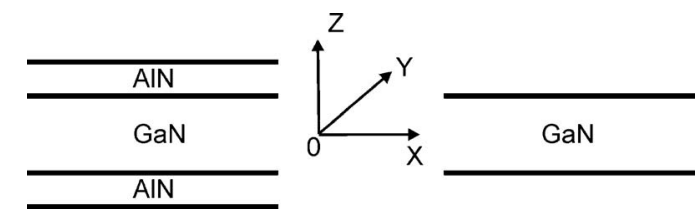

Fig. 1. Schematics of a free-standing AlN/GaN/AlN heterostructure (left) and a free-standing GaN thin film (right). The coordinate system is shown in the middle.

distribution, energy, and group velocity. An important term in Eq. (1) is the phonon dispersion relation $\omega_{\alpha}(\mathbf{k})$, which describes the dependence of the phonon angular frequency on the wave vector.

We model the thermal conductivity in a semiconductor nanostructure as follows: ${ }^{9-11}$ (1) Find the phonon distribution by solving the phonon Boltzmann transport equation in the relaxation-time approximation; ${ }^{9}$ (2) derive the phonon dispersion by solving the continuum elastic wave equation with the appropriate boundary conditions; (3) calculate the phonon group velocity $\mathbf{V}=d \omega / d \mathbf{k}$; and (4) replace the summation in Eq. (1) by an integral over the phonon density of states.

A generalized expression for the lattice thermal conductivity can be derived using the relation $\kappa=J /|\nabla T|$. The phonon dispersion plays an important role in determining the lattice thermal conductivity through its effects on other phonon properties, such as the group velocity and density of states.

In the following we discuss the numerical calculation of phonon dispersion in two types of nanostructures: a freestanding thin film and a three-layer heterostructure. We follow a general approach outlined by Balandin and co-workers. ${ }^{12}$ The derivation and calculation of the lattice thermal conductivity in a thin film and heterostructure can be found in Ref. 13.

\section{CONTINUUM ELASTIC WAVE EQUATIONS}

We consider a free-standing $\mathrm{GaN}$ thin film and a freestanding $\mathrm{AlN} / \mathrm{GaN} / \mathrm{AlN}$ heterostructure (see Fig. 1). The phrase "free-standing" describes an interface between a semiconductor and a vacuum. A similar situation is that of a string with one end that is free to move. A thin film refers to a slab of material with a confined direction along the film thickness. The AlN/GaN/AlN heterostructure is a threelayer structure, with a core layer embedded between two cladding layers made of a different material. Figure 1 shows the schematics of the two structures. The $x$ and $y$ directions in the plane of the structures are unbounded, and the direction along the $z$ axis is confined. Both structures represent quasi-two-dimensional systems, where the lattice wave propagates in the $(x, y)$ plane. For example, we consider a shear wave traveling in the $x$ direction with a particle displacement in the $y$ direction. In this direction, the continuum elastic wave equation is given by ${ }^{4}$

$$
\rho \frac{\partial^{2} U_{y}}{\partial t^{2}}=\frac{\partial Y_{x}}{\partial x}+\frac{\partial Y_{y}}{\partial y}+\frac{\partial Y_{z}}{\partial z},
$$

where $\rho$ is the density and $U_{y}$ is the $y$ component of the displacement vector. $Y_{x}, Y_{y}$, and $Y_{z}$ are the stress components. For example, $Y_{x}$ represents a force per unit area in the $y$ direction acting on a plane with its normal in the $x$ direc- tion. According to Hooke's law, if the deformations are small, the stress components are linearly related to the strain components; the coefficients are called the elastic stiffness constants. In a hexagonal crystal, such as wurtzite $\mathrm{GaN}$, the independent elastic stiffness constants are $C_{11}, C_{12}, C_{13}, C_{33}$, $\mathrm{C}_{44}$, and $\mathrm{C}_{66}{ }^{14}$

We look for solutions to Eq. (2) of the form of a sinusoidal wave,

$$
U_{y}=u_{y}(z) \exp [i(\omega t-k x)]
$$

where $u_{y}$ is the amplitude, which depends on the $z$ coordinate, and $k$ is the $x$ component of the wave vector. If we substitute Eq. (3) into Eq. (2) and use the relations between the stress, strain, and displacement components, as described in Ref. 4, we derive the elastic wave equations for a $\mathrm{GaN}$ thin film and an AlN/GaN/AlN heterostructure. The derivation is straightforward, and we leave it as an exercise. The results are given in Eqs. (4) and (7) for a GaN thin film and an $\mathrm{AlN} / \mathrm{GaN} / \mathrm{AlN}$ heterostructure, respectively.

For a GaN thin film, Eq. (2) can be simplified to the second-order ordinary differential equation

$$
\frac{d^{2} u_{y}(z)}{d z^{2}}+\frac{1}{C_{44, \mathrm{GaN}}}\left(\rho_{\mathrm{GaN}} \omega^{2}-C_{66, \mathrm{GaN}} k^{2}\right) u_{y}(z)=0 .
$$

The free-standing boundary conditions require that the stress and strain are zero at the surfaces, that is,

$$
\left.\frac{d u_{y}(z)}{d z}\right|_{z= \pm t / 2}=0
$$

where $t$ is the thickness of the thin film. The boundary-value problem represented by Eq. (4) with the free-standing boundary conditions can be solved analytically.

A three-layer heterostructure is different because it is inhomogeneous along the $z$ direction, and therefore the material parameters, such as $\rho$ and $C_{i i}(i=4$ or 6$)$, depend on the $z$ coordinate. We define the material parameters as piecewise functions of $z$ as

$$
\rho(z)= \begin{cases}\rho_{\mathrm{AlN}}, & -t(\mathrm{GaN}) / 2-t(\mathrm{AlN}) \leqslant z<-t(\mathrm{GaN}) / 2, \\ \rho_{\mathrm{GaN}}, & -t(\mathrm{GaN}) / 2 \leqslant z<t(\mathrm{GaN}) / 2 \\ \rho_{\mathrm{AlN}}, & t(\mathrm{GaN}) / 2 \leqslant z \leqslant t(\mathrm{GaN}) / 2+t(\mathrm{AlN}) .\end{cases}
$$

The elastic stiffness constants $C_{44}$ and $C_{66}$ can be defined similarly. The elastic wave equation for the heterostructure is given by

$$
\begin{aligned}
\frac{d^{2} u_{y}(z)}{d z^{2}} & +\frac{1}{C_{44}(z)} \frac{d C_{44}(z)}{d z} \frac{d u_{y}(z)}{d z} \\
& +\frac{1}{C_{44}(z)}\left[\rho(z) \omega^{2}-C_{66}(z) k^{2}\right] u_{y}(z)=0 .
\end{aligned}
$$

Note that the boundary conditions at the GaN-AIN interface have been implicitly included in the $z$-dependent material parameters. These boundary conditions are different from those at the GaN-vacuum interface as in a free-standing GaN thin film. This inhomogeneous medium approach has been used in acoustics to analyze wave propagation in composite plates. ${ }^{15}$ At the outer surfaces of the heterostructure, freestanding boundary conditions apply. Equation (7) with the free-standing boundary conditions cannot be solved analytically and numerical techniques need to be applied. Note that 
for a thin film, $C_{44}$ is independent of $z$ and $d C_{44}(z) / d z$ vanishes. Equation (7) is then reduced to Eq. (4).

\section{NUMERICAL SOLUTION OF PHONON DISPERSION}

The phonon dispersion for a shear wave in a thin film can be solved analytically. The treatment is similar to a standing wave on a string and a particle in a box problem in quantum mechanics. The solution is

$$
\omega=\sqrt{\frac{C_{66} k^{2}+C_{44} k_{z}^{2}}{\rho},}
$$

where $k_{z}$ is the component of the wave vector in the $z$ direction. Due to the boundary conditions, $k_{z}$ is quantized and is given by $k_{z}=n \pi / t$, where $n$ is a positive integer and $t$ is the thickness of the thin film. The quantization of the phonon modes is due to the spatial confinement of phonons, similar to the confinement of an electron in a quantum well.

To solve for the phonon dispersion in a heterostructure numerically, we use a finite difference method and follow a general strategy in vibration analysis. ${ }^{16}$ Students can also apply the finite difference method to a thin film and compare the numerical solution with the analytical one. The numerical algorithm takes four steps.

(1) Divide the total thickness $t$ of the heterostructure into $N$ equal parts of width $h=t / N$. The $z$ coordinate of each node is given by: $z_{i}=z_{0}+i h$, where $z_{0}=-t / 2$, and $i=0,1, \ldots, N$.

(2) Apply the central-difference formula of the finitedifference approximation to the first and second-order derivatives in Eq. (7):

$$
\frac{d^{2} u}{d z^{2}} \approx \frac{\left(u_{i+1}-2 u_{i}+u_{i-1}\right)}{h^{2}} \text { and } \frac{d u}{d z} \approx \frac{\left(u_{i+1}-u_{i-1}\right)}{2 h} .
$$

For simplicity, the subscript $y$ is omitted. We apply Eq. (9) to Eq. (7) at each node $i$ and obtain

$$
\begin{gathered}
\left(\frac{-C_{44, i+1}+4 C_{44, i}+C_{44, i-1}}{4 h^{2}}\right) u_{i-1}+\left(\rho_{i} \omega^{2}-\frac{2 C_{44, i}}{h^{2}}\right. \\
\left.-C_{66, i} k^{2}\right) u_{i}+\left(\frac{C_{44, i+1}+4 C_{44, i}-C_{44, i-1}}{4 h^{2}}\right) u_{i+1}=0 .
\end{gathered}
$$

Equation (10) represents $N+1$ algebraic equations with $i$ $=0,1, \ldots, N$. If we apply the free-standing boundary conditions and use the central-difference approximation, we find

$$
\begin{aligned}
\left.\frac{d u(z)}{d z}\right|_{z=-t / 2} & \approx \frac{\left(u_{1}-u_{-1}\right)}{2 h}=0 \text { and }\left.\frac{d u}{d z}\right|_{z=t / 2} \\
& \approx \frac{\left(u_{N+1}-u_{N-1}\right)}{2 h}=0,
\end{aligned}
$$

which gives $u_{-1}=u_{1}$ and $u_{N+1}=u_{N-1}$.

(3) Convert Eq. (10) into a matrix eigenvalue problem of the general form $[A] \mathbf{u}=\lambda[B] \mathbf{u}$, where $[A]$ and $[B]$ are both $(N+1) \times(N+1)$ coefficient matrices, $\lambda=\omega^{2}$ is the eigenvalue, and $\mathbf{u}=\left[u_{0}, u_{1}, \ldots, u_{N}\right]^{T}$ is the eigenvector. We can show that $[B]$ is also a diagonal matrix. To derive the matrix eigenvalue form, we suggest that students follow three steps. First, write out Eq. (10) at each node $i$ and replace $u_{-1}$ and $u_{N+1}$ by $u_{1}$ and $u_{N-1}$, respectively, as given in Eq. (11). Second, note that
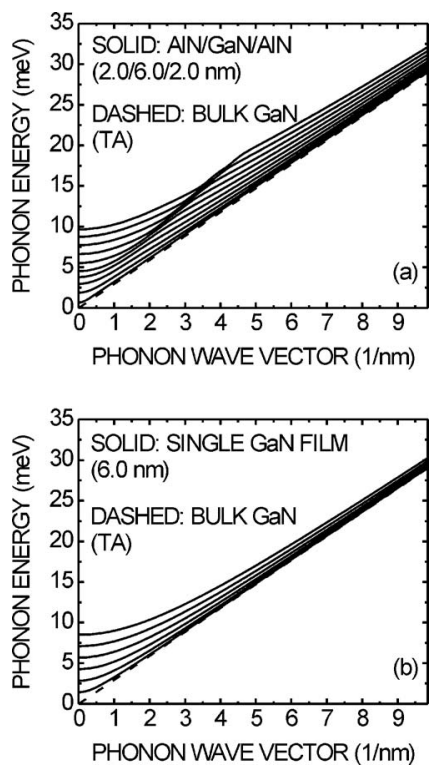

Fig. 2. Acoustic phonon dispersion relations for the shear modes in (a) a free-surface AlN/GaN/AIN heterostructure with a core layer thickness of $6 \mathrm{~nm}$ and a cladding layer thickness of $2 \mathrm{~nm}$, and (b) a free-surface single GaN thin film with a thickness of $6 \mathrm{~nm}$. The dashed line shows the dispersion relation for the transverse acoustic (TA) waves in bulk GaN. Used with permission from Ref. 13. (C) 2006, American Institute of Physics.

the problem is now represented by a system of $(N+1)$ linear and homogeneous equations with $(N+1)$ unknowns, $u_{0}, u_{1}, \ldots, u_{N}$. Then convert this system of linear equations into matrix form and transform it to an eigenvalue problem. Following the approach in Ref. 16, we then transform the general eigenvalue problem to the standard form: $[P] \mathbf{X}$ $=\lambda \mathbf{X}$. It is straightforward to show that $[P]=[C]^{-1}[A][C]^{-1}$, $\mathbf{X}=[C] \mathbf{u}$, and $[C]$ is a diagonal matrix with elements given by $c_{i i}=b_{i i}^{1 / 2}$.

(4) Finally the algorithm is translated into a computer code written in MATLAB ${ }^{\circ}$. The eigenvalues $\lambda$, are solved for each value of the wave vector $k$ using the intrinsic function eig. The corresponding angular frequencies are calculated from $\omega=\lambda^{1 / 2}$. The phonon dispersion is then plotted as a function of $\omega$ versus $k$.

\section{SIMULATION RESULTS AND DISCUSSIONS}

We performed simulations for a free-standing $6.0 \mathrm{~nm} \mathrm{GaN}$ thin film and a free-standing 2.0/6.0/2.0 nm AlN/GaN/AlN heterostructure. The results are shown in Fig. 2. Note that the vertical axis is the phonon energy, which differs from the phonon angular frequency by a factor of $\hbar$. The dashed line in Fig. 2 shows the phonon dispersion in bulk GaN, which shows the linear dependence of the angular frequency on wave vector, as expected from the elastic continuum approximation. Note that phonon dispersion in the nanostructures has different characteristics from that in the bulk. The angular frequency has a set of discrete values for each wave vector, resulting in discrete phonon branches. The origin of the quantization of the phonon dispersion is the spatial confinement of the phonons in the $z$ direction, which leads to the quantization of $k_{z}$. In Fig. 3 we show a plot of the angular 


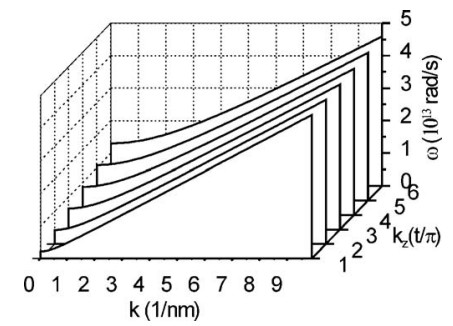

Fig. 3. Plot of the angular frequency $\omega$ as a function of the $x$ and $z$ components of the wave vector, $k$ and $k_{z}$, for the shear modes in a $6 \mathrm{~nm} \mathrm{GaN}$ thin film. The quantization of $k_{z}$ and the resulting discrete phonon branches can be clearly seen. In the plot, $k_{z}$ is measured in units of $\pi / t$, where $t$ is the thickness of the GaN thin film.

frequency as a function of both $k$ and $k_{z}$ in a thin film. The quantization of $k_{z}$ and the resulting discrete phonon branches can be clearly seen.

The difference in the phonon dispersion of the two nanostructures can be explained in terms of the different boundary conditions at the $\mathrm{GaN}$ interface. In a free-standing $\mathrm{GaN}$ thin film, the elastic waves experience complete reflection at the GaN-vacuum surface. The situation is similar to the reflection of a traveling pulse at the free end of a string. In contrast, in an AlN/GaN/AlN heterostructure, the GaN layer is embedded within another medium, AlN, which has a dissimilar density and elastic stiffness constants. This situation is similar to a heavier string attached to a lighter string. The elastic waves now undergo partial reflection and partial transmission at the GaN-AlN interface. The difference in the boundary conditions at the GaN interface leads to different characteristics of the phonon dispersion in the two nanostructures. Because the boundary conditions can be modified by adjusting the parameters of the interfacing materials, such as the elastic properties and thickness, it is possible to tune the phonon dispersion. As mentioned in Sec. I, the idea of phonon engineering opens up new and exciting opportunities for materials engineering. ${ }^{3}$

\section{ACKNOWLEDGMENTS}

The author would like to thank students Xavier Lange and Chris Richardson for their participation in this project, and the Council on Faculty Research at Eastern Illinois University for financially supporting this research.

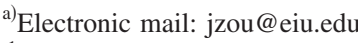

${ }^{1}$ S. Bandyopadhyay, N. Kouklin, and L. Menon, "Device and circuit applications of electrochemically self-assembled quantum dots and wires," in Quantum Dots and Nanowires, edited by S. Bandyopadhyay and H. S. Nalwa (American Scientific Publishers, Los Angeles, CA, 2003), Chap. 9.

${ }^{2}$ G. Chen, "Phonon heat conduction in nanostructures," Int. J. Therm. Sci. 39, 471-480 (2000).

${ }^{3}$ A. A. Balandin, "Nanophononics: Phonon engineering in nanostructures and nanodevices," J. Nanosci. Nanotechnol. 5, 1-8 (2005).

${ }^{4}$ C. Kittel, Introduction to Solid State Physics (John Wiley \& Sons, New York, 1996), 7th ed., pp. 99-107; 80-88.

${ }^{5}$ A. Khitun, A. Balandin, and K. L. Wang, "Modification of the lattice thermal conductivity in silicon quantum wires due to spatial confinement of acoustic phonons," Superlattices Microstruct. 26, 181-193 (1999).

${ }^{6}$ N. Bannov, V. Aristov, V. Mitin, and M. A. Stroscio, "Electron relaxation times due to the deformation-potential interaction of electrons with confined acoustic phonons in a free-standing quantum well," Phys. Rev. B 51, 9930-9942 (1995).

${ }^{7}$ S. Yu, K. W. Kim, M. A. Stroscio, and G. J. Iafrate, "Electron-acousticphonon scattering rates in cylindrical quantum wires," Phys. Rev. B 51, 4695-4698 (1995).

${ }^{8}$ R. A. Serway and J. W. Jewett, Physics for Scientists and Engineers with Modern Physics (Thomson-Brooks/Cole, Belmont, CA, 2004), 6th ed., p. 624.

${ }^{9}$ C. M. Bhandari and D. M. Rowe, Thermal Conduction in Semiconductors (John Wiley \& Sons, New York, 1988), pp. 83; 107-109.

${ }^{10}$ A. A. Balandin and K. L. Wang, "Significant decrease of the lattice thermal conductivity due to phonon confinement in a free-standing semiconductor quantum well," Phys. Rev. B 58, 1544-1549 (1998).

${ }^{11} \mathrm{~J}$. Zou and A. Balandin, "Phonon heat conduction in a semiconductor nanowire," J. Appl. Phys. 89, 2932-2938 (2001).

${ }^{12}$ A. A. Balandin, D. L. Nika, and E. P. Pokatilov, "Phonon spectrum and group velocities in wurtzite hetero-structures," Phys. Status Solidi C 1, 2658-2661 (2004).

${ }^{13}$ J. Zou, X. Lange, and C. Richardson, "Lattice thermal conductivity of nanoscale AlN/GaN/AlN heterostructures: Effects of partial phonon spatial confinement," J. Appl. Phys. 100, 104309-1-8 (2006).

${ }^{14}$ R. E. Newnham, Properties of Materials: Anisotropy, Symmetry, Structure (Oxford University Press, New York, 2005), p. 252.

${ }^{15}$ B. A. Auld, Acoustic Fields and Waves in Solids (Krieger, Malabar, FL, 1990), 2nd ed., Vol. 2, pp. 114-117.

${ }^{16}$ S. S. Rao, Applied Numerical Methods for Engineers and Scientists (Prentice Hall, Upper Saddle River, NJ, 2002), pp. 280-285; 754-761. 